\title{
Atorvastatin alleviates iodinated contrast media-induced cytotoxicity in human proximal renal tubular epithelial cells
}

\author{
GAI-LING LIU ${ }^{1,2 *}$, RONG LEI $^{1 *}$, SHAO-BIN DUAN $^{1}$, MI-MI TANG $^{3}$, MIN LUO $^{1}$ and QIAN XU $^{1}$ \\ ${ }^{1}$ Department of Nephrology, The Second Xiangya Hospital of Central South University, Changsha, Hunan 410011; \\ ${ }^{2}$ Department of Nephrology and Rheumatology, Henan Provincial People's Hospital, Zhengzhou, Henan 450003; \\ ${ }^{3}$ Institute of Clinical Pharmacy and Pharmacology, The Xiangya Hospital of \\ Central South University, Changsha, Hunan 410008, P.R. China
}

Received June 28, 2016; Accepted April 21, 2017

DOI: $10.3892 /$ etm.2017.4859

\begin{abstract}
Contrast media (CM)-induced nephropathy (CIN) is a serious complication of intravascularly applied radiocontrast media. At present, no drugs have been approved for the prevention of CIN. The present study aimed to explore the effects and potential mechanisms of atorvastatin on iodinated CM-induced cytotoxicity in the human proximal renal tubular epithelial cells. The cytotoxic effect of iohexol (50,100 and $200 \mathrm{mg} \mathrm{I} /$ $\mathrm{ml})$ and the protective effect of atorvastatin pretreatment $(1$, 20 and $40 \mu \mathrm{M}$ ) were assessed. The cytotoxicity of iohexol was evaluated via the MTT cell viability and lactate dehydrogenase assays. The amount of apoptotic cells was determined by flow cytometry. Morphological changes in HK-2 cells were observed via transmission electron microscopy. The mRNA expression of NOX4 and p22phox was measured through reverse transcription-quantitative polymerase chain reaction analysis. The cytotoxicity was induced by iohexol in HK-2 cells. Atorvastatin was identified to significantly alleviate the suppression of cell viability induced by iohexol. Notably, $40 \mu \mathrm{M}$ atorvastatin also significantly reduced the mRNA expression of intracellular NOX4 and p22phox, and the percentage of apoptotic cells. Furthermore, morphological changes characteristic of injured cells were alleviated by atorvastatin pretreatment. These results suggest that atorvastatin exhibits a protective effect on HK-2 cells against iohexol-induced cytotoxicity through the downregulation of NOX4 and p22phox. Thus, atorvastatin is a potential therapeutic agent for the prevention of CIN and required further study.
\end{abstract}

Correspondence to: Professor Shao-Bin Duan, Department of Nephrology, The Second Xiangya Hospital of Central South University, 139 Renmin Road, Changsha, Hunan 410011, P.R. China E-mail: duansb528@qq.com

*Contributed equally

Key words: contrast media-induced nephropathy, acute kidney injury, oxidative stress, nicotinamide adenine dinucleotide phosphate oxidase, atorvastatin

\section{Introduction}

With advancements in imaging and interventional technologies, iodinated contrast media (CM) is widely applied for enhancing the visibility of vascular structures and organs during radiographic procedures. Iodinated $\mathrm{CM}$-induced nephropathy (CIN) is the third most common cause of hospital-acquired acute kidney injury, which is associated with a lengthened hospital stay, increased health care costs and higher risk of mortality (1-3). Therefore, it is of great importance to explore effective strategies to prevent CIN. There is broad consensus that volume expansion could reduce the risk of CIN (2); however, no drugs have been approved for the prevention of CIN.

Nicotinamide adenine dinucleotide phosphate oxidase (NOX) is a source of reactive oxygen species (ROS) and can therefore be used as an indicator of the oxidative status of cells, and as a marker of renal tubular epithelial cell and vascular endothelial cell damage (4). Among all members of the NOX family, NOX4 results in the highest hydrogen peroxide to superoxide ratio (5). Upregulation of NOX4 protein expression is associated with increased oxidative stress. p22 phagocyte B-cytochrome (p22phox) interacts with NOX4 and enhances its activity (6). Inhibition of p22phox serves a protective role in cells by inhibiting the activation of NOX4 and subsequent generation of ROS (6).

Statins, in addition to their impact on blood cholesterol levels, have been identified to have multiple pleiotropic effects, including improving endothelial function, and decreasing oxidative stress and inflammation $(7,8)$. A number of retrospective and prospective studies have demonstrated the potential protective effect of statins on CIN (9-11). In addition, statins have been reported to exhibit antioxidant effects through decreasing the mRNA expression of NOX4 and p22phox $(12,13)$. These findings suggest that statins may exhibit a protective effect in CIN through decreasing the expression of NOX4 and p22phox in kidney cells.

\section{Materials and methods}

Cell culture. Human proximal tubular epithelial HK-2 cells were obtained from the American Type Culture Collection 
(Manassas, VA, USA). The cells were cultured at $37^{\circ} \mathrm{C}$ in a humidified atmosphere with $5 \% \mathrm{CO}_{2}$. The culture medium, Dulbecco's modified Eagle's medium/Nutrient Mixture F-12 (DMEM/F12; Gibco; Thermo Fisher Scientific, Inc., Waltham, MA, USA), was supplemented with $10 \%$ heat-inactivated fetal calf serum (FCS; Gibco; Thermo Fisher Scientific, Inc.), 2 mM glutamine (Gibco; Thermo Fisher Scientific, Inc.), 100 IU/ml penicillin and $100 \mu \mathrm{g} / \mathrm{ml}$ streptomycin. The cells were grown as monolayers in 24- or 96-well plates. Cells were passaged via digestion with $0.25 \%$ trypsin when they reached $70-80 \%$ confluence. Iohexol (Shanghai TongYong Pharmaceutical Co., Ltd., Shanghai, China) was used as the ionic low-osmolality radiocontrast agent. Atorvastatin was supplied by Guangzhou Nucien Pharmaceutical Co., Ltd. (Guangzhou, China).

Establishment of a CIN cell model. To evaluate the cytotoxicity of CM to HK-2 cells, the cells were cultured in different concentrations $(50,100$ or $200 \mathrm{mg} \mathrm{I} / \mathrm{ml})$ of iohexol dissolved in DMEM/F12 with $1 \%$ FCS for 3, 6 or $12 \mathrm{~h}$. The appropriate concentration of iohexol and duration of treatment for the cell model was chosen based on the MTT and cell injury assay results.

Groupings. The four groups used for the experiments were as follows: Group A, the untreated control group; group B, the cell injury group, which was treated with iohexol $(200 \mathrm{mgI} / \mathrm{ml})$ for $6 \mathrm{~h}$; group C, the atorvastatin pretreatment group, which was treated with atorvastatin (group $\mathrm{C} 1,1 \mu \mathrm{M}$; group $\mathrm{C} 2$, $20 \mu \mathrm{M}$; group $\mathrm{C} 3,40 \mu \mathrm{M}$ ) for $2 \mathrm{~h}$ prior to iohexol treatment (200 $\mathrm{mgI} / \mathrm{ml}$ ); and group $\mathrm{D}$, the atorvastatin alone group, which was treated with $40 \mu \mathrm{M}$ atorvastatin for $2 \mathrm{~h}$.

MTT cell viability assay. The MTT assay was used to assess the HK-2 cell viability, as previously described (14). Briefly, HK-2 cells were seeded in a 96-wells plate with $1 \times 10^{5}$ cells $/ \mathrm{ml}$ per well, with six wells for each group. Prior to treatment, the confluency of cells reached to $70-80 \%$. Confluent HK-2 cell monolayers were incubated for 3,6 or $12 \mathrm{~h}$ with control medium or different concentrations of iodinated CM $(50,100$ or $200 \mathrm{mg} \mathrm{I} / \mathrm{ml}$ ). MTT reagent (5 mg I/ml; $20 \mu \mathrm{l})$ was then added to the plates, which were incubated for $4 \mathrm{~h}$ in $37^{\circ} \mathrm{C}$, after which $150 \mu \mathrm{l}$ dimethyl sulfoxide was added and the plates were oscillated for $10 \mathrm{~min}$. Cell viability was measured at $490 \mathrm{~nm}$ using a Labsystems Dragon Wellscan MK2 microplate reader (Labsystems Diagnostics Oy, Vantaa, Finland).

Cell injury assay. HK-2 cells were seeded in 24-well plates at a density of $1-5 \times 10^{5}$ cells $/ \mathrm{ml}$. When $70-80 \%$ confluency was reached, the cells were incubated with DMEM/F12 medium for $12 \mathrm{~h}$ in $37^{\circ} \mathrm{C}$ and treated with iohexol $(50,100$ or $200 \mathrm{mg} \mathrm{I} /$ $\mathrm{ml}$ ) for 3, 6 or $12 \mathrm{~h}$. Cells injury was assessed using the lactate dehydrogenase assay (LDH; Sangon Biotech Co., Ltd., Shanghai, China) using an automatic biochemistry analyzer (7170A; Hitachi Ltd., Tokyo, Japan).

Apoptosis assay. HK-2 cells were plated in 6-well plates at a density of $5 \times 10^{4}$ cells/well with DMEM/F12 supplemented with $10 \%$ FCS. Then, the medium was replaced with medium without FCS for $24 \mathrm{~h}$. The cells were then incubated in accordance with the requirements of each group as described above.
Subsequently, the cells were washed with PBS and harvested by trypsinization. Then, the cells were washed twice with 1X Annexin $\mathrm{V}$ binding buffer and resuspended in binding buffer. Annexin V-FITC was then added and the plates were incubated in the dark for $15 \mathrm{~min}$ at room temperature. Finally, propidium iodide (PI) staining solution was added and the plates were incubated in the dark for another $5 \mathrm{~min}$ at room temperature, these components were from an Annexin V FITC apoptosis detection kit (100TST; BD Biosciences, Franklin Lakes, NJ, USA), which was used according to the manufacturer's protocol. The percentage of apoptotic was determined using the FACScan flow cytometer followed by analysis with BD CellQuest Pro software (BD Biosciences).

Transmission electron microscopy examination. Confluent HK-2 cells from each group were incubated in a $25 \mathrm{~cm}^{2}$ culture flask, then cells were harvested and fixed with Karnovsky fixative for $16 \mathrm{~h}$ at $4^{\circ} \mathrm{C}$. The cells were processed by ultramicrotomy (50-100 nm sections), double stained with lead citrate and uranyl acetate, and observed under an EM 300 electron microscope (Philips, Amsterdam, The Netherlands).

Reverse transcription-quantitative polymerase chain reaction (RT-qPCR) analysis. Total RNA was extracted using TRIzol reagent (Invitrogen; Thermo Fisher Scientific, Inc.). RT was performed using a High-Capacity cDNA Reverse Transcription kit (Takara Biotechnology Co., Ltd., Dalian, China) according to the manufacturer's protocol. cDNA was synthesized from $2 \mu \mathrm{g}$ total RNA, total cDNA $(1 \mu \mathrm{l})$ was loaded in each well and qPCR was performed using an Applied Biosystems 7300 Real-Time RCR system and SYBR-Green PCR Master mix (both Thermo Fisher Scientific, Inc.) according to the manufacturer's protocol. The qPCR thermocycling conditions were as follows: $95^{\circ} \mathrm{C}$ for $10 \mathrm{~min}$; followed by 40 cycles of $95^{\circ} \mathrm{C}$ for $15 \mathrm{sec}$ and $60^{\circ} \mathrm{C}$ for $1 \mathrm{~min}$. The qPCR primers are provided in Table I and were obtained from Sangon Biotech Co., Ltd. mRNA levels were quantified using the $2^{-\Delta \Delta \mathrm{Cq}}$ method (15), where $\Delta \mathrm{Cq}$ is the result of the quantification cycle $(\mathrm{Cq})$ of the treated sample subtracted from the $\mathrm{Cq}$ value of the untreated or 0 time point control sample. The relative amount of mRNA was normalized to GAPDH.

Statistical analysis. Results are expressed as the mean \pm standard deviation. The statistical significance of differences between more than two groups was assessed by one-way analysis of variance followed by a post hoc Fisher's least significant difference test. SPSS software (version 17.0; IBM Corp., Armonk, NY, USA) was used for all statistical analysis. $\mathrm{P}<0.05$ was considered to indicate a statistically significant difference.

\section{Results}

Iodinated CM induces cytotoxicity in HK-2 cells. The cytotoxicity induced by the iodinated contrast agent iohexol in HK-2 cells was evaluated using the MTT cell viability assay and LDH cell injury assays. As demonstrated in Tables II and III, 100 and $200 \mathrm{mg} \mathrm{I} / \mathrm{ml}$ iohexol significantly decreased HK-2 cell viability and induced HK-2 cell injury in time- and concentration-dependent manner compared with the control 
Table I. Quantitative polymerase chain reaction primer sequences.

Primer direction $\left(5^{\prime}-3^{\prime}\right)$

Target mRNA

NOX4

p22phox

GADPH
Sense

CTCAGCGGAATCAATCAGCTGTG

CAGTGGTACTTTGGTGCCTACTCC

AGAAGGCTGGGGCTCATTTG
Antisense

AGAGGAACACGACAATCAGCCTTAG

GGTGGAGCCCTTCTTCCTCT

AGGGGCCATCCACAGTCTTC

p22phox, p22 phagocyte B-cytochrome; NOX, nicotinamide adenine dinucleotide phosphate oxidase.

Table II. Iohexol decreases HK-2 cell viability in a time- and dose-dependent manner ( $\mathrm{n}=6)$.

\begin{tabular}{llll}
\hline & & MTT assay (OD value) \\
\cline { 2 - 4 } Treatment group & 3 h treatment & 6 h treatment & $12 \mathrm{~h} \mathrm{treatment}$ \\
\hline Control & $0.587 \pm 0.003$ & $0.598 \pm 0.005$ & $0.610 \pm 0.004$ \\
Iohexol $(50 \mathrm{mg} \mathrm{I} / \mathrm{ml})$ & $0.519 \pm 0.002$ & $0.462 \pm 0.006$ & $0.412 \pm 0.009^{\mathrm{a}}$ \\
Iohexol $(100 \mathrm{mg} \mathrm{I} / \mathrm{ml})$ & $0.422 \pm 0.001^{\mathrm{a}}$ & $0.408 \pm 0.003^{\mathrm{a}}$ & $0.363 \pm 0.009^{\mathrm{a}}$ \\
Iohexol $(200 \mathrm{mg} \mathrm{I} / \mathrm{ml})$ & $0.377 \pm 0.009^{\mathrm{b}}$ & $0.347 \pm 0.001^{\mathrm{b}}$ & $0.267 \pm 0.005^{\mathrm{b}}$
\end{tabular}

${ }^{a} \mathrm{P}<0.05$ and ${ }^{b} \mathrm{P}<0.01$ vs. the control group at the same time point. OD, optical density.

Table III. Iohexol increases HK-2 cell LDH levels $(n=6)$.

LDH level (U/1)

\begin{tabular}{lccc}
\cline { 2 - 3 } Treatment group & $3 \mathrm{~h}$ treatment & $6 \mathrm{~h}$ treatment & $12 \mathrm{~h}$ treatment \\
\hline Control & $19 \pm 1.23$ & $29 \pm 2.15$ & $50 \pm 1.4$ \\
Iohexol $(50 \mathrm{mg} \mathrm{I} / \mathrm{ml})$ & $22 \pm 2.6$ & $35 \pm 2.6$ & $59 \pm 1.9$ \\
Iohexol $(100 \mathrm{mg} \mathrm{I} / \mathrm{ml})$ & $36 \pm 2.1^{\mathrm{a}}$ & $50 \pm 3.3^{\mathrm{a}}$ & $98 \pm 2.9^{\mathrm{b}}$ \\
Iohexol $(200 \mathrm{mg} \mathrm{I} / \mathrm{ml})$ & $49 \pm 1.9^{\mathrm{a}}$ & $71 \pm 3.21^{\mathrm{b}}$ & $130 \pm 2.5^{\mathrm{b}}$ \\
\hline
\end{tabular}

${ }^{\mathrm{a}} \mathrm{P}<0.05$ and ${ }^{\mathrm{b}} \mathrm{P}<0.01$ vs. the control group at the same time point. $\mathrm{LDH}$, lactate dehydrogenase.

group $(\mathrm{P}<0.05)$. Thus, HK-2 cell treated with $200 \mathrm{mg} \mathrm{I} / \mathrm{ml}$ iohexol for $6 \mathrm{~h}$ were used as the injured cell model for further analysis.

Atorvastatin attenuates the iohexol-induced suppression of $H K-2$ cell viability. To evaluate the protective effect of atorvastatin on iohexol-induced cytotoxicity in HK-2 cells, the viability of the injured cell model pretreated with different concentrations of atorvastatin $(1,20$ or $40 \mu \mathrm{M})$ for $2 \mathrm{~h}$ was evaluated. As shown in Table IV, atorvastatin significantly attenuated iohexol-induced HK-2 cell cytotoxicity in a dose-dependent manner compared with the control and/or model groups $(\mathrm{P}<0.05)$. Thus, $\mathrm{HK}-2$ cells pretreated with $40 \mu \mathrm{M}$ atorvastatin were used for further analysis.

Atorvastatin inhibits iohexol-induced HK-2 cell apoptosis. As demonstrated in Fig. 1, iohexol significantly induced the apoptosis of HK-2 cells compared with the control group $(\mathrm{P}<0.05)$. Pretreatment with different concentrations of atorvastatin significantly reduced this increase in apoptosis compared with the control and/or cell injury groups $(\mathrm{P}<0.05$; Fig. 1). Pretreatment with $40 \mu \mathrm{M}$ atorvastatin was the optimal concentration to inhibit the iohexol-induced apoptosis. These observations were confirmed by flow cytometry with Annexin V/PI double-staining (data not shown).

Atorvastatin alleviates iohexol-induced HK-2 cell morphological changes. Transmission electron microscopy was used to observe the ultrastructure of the cells. The nuclear membrane of HK-2 cells in the control group was clear and continuous, and the nuclei contained uniform chromatin (Fig. 2A). However, the morphological characteristics of apoptosis, including nuclear fragmentation, organelle reduction and mitochondrial vacuolar degeneration, were frequently 
Table IV. Atorvastatin attenuates iohexol-induced suppression of HK-2 cell viability $(n=6)$.

\begin{tabular}{lc}
\hline Group & MTT assay (OD value) \\
\hline A & $0.601 \pm 0.036$ \\
B & $0.290 \pm 0.022^{\mathrm{a}}$ \\
C1 & $0.342 \pm 0.007^{\mathrm{b}}$ \\
C2 & $0.358 \pm 0.002^{\mathrm{b}, \mathrm{c}}$ \\
C3 & $0.439 \pm 0.006^{\mathrm{b}, \mathrm{c}}$ \\
D & $0.596 \pm 0.027$ \\
\hline
\end{tabular}

${ }^{\mathrm{a}} \mathrm{P}<0.05$ and ${ }^{\mathrm{b}} \mathrm{P}<0.01$ vs. group $\mathrm{A} ;{ }^{\mathrm{c}} \mathrm{P}<0.05$ vs. group $\mathrm{B}$.

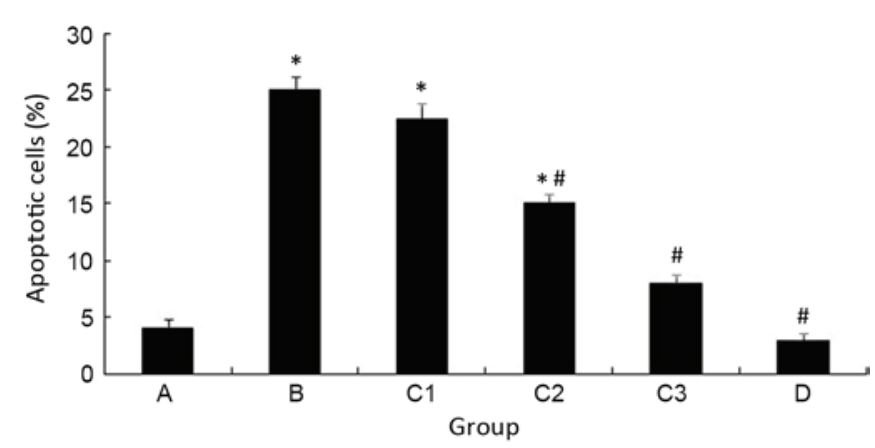

Figure 1. Atorvastatin inhibits iohexol-induced HK-2 cell apoptosis. " $\mathrm{P}<0.05$ vs. group $\mathrm{A} ;{ }^{\#} \mathrm{P}<0.05$ vs. group $\mathrm{B}$.

detected in the cell injury group (Fig. 2B). Notably, the nuclear membrane in the atorvastatin pretreatment group remained continuous (Fig. 2C). Perinuclear expansion, partial nuclei and chromatin margination were also observed (Fig. 2C). The morphology and structure of the majority of the mitochondria remained normal, with only partial vacuolar degeneration observed (Fig. 2C).

Atorvastatin inhibits iohexol-induced NOX subunit expression in HK-2 cells. The expression of NOX4 and p22phox mRNA was measured in the present study using RT-qPCR (Fig. 3). When HK-2 cells were treated with iohexol for $6 \mathrm{~h}$, the mRNA levels of NOX4 and p22phox were significantly increased compared with the control group $(\mathrm{P}<0.05)$. However, pretreatment with atorvastatin $(40 \mu \mathrm{M})$ significantly attenuated the iohexol-induced expression of NOX4 and 22 phox $(\mathrm{P}<0.05)$.

\section{Discussion}

The results of the present study demonstrated atorvastatin could protect HK-2 cells from iohexol-induced cytotoxicity, through attenuating the inhibition of cell viability, inhibiting apoptosis and the downregulating mRNA expression of NOX4 and p22phox.

CIN is a serious clinical complication of imtravascularly applied iodinated CM (16). The present study and a previous study (17) have demonstrated that iodinated CM induces cell injury significantly in time- and concentration-dependent
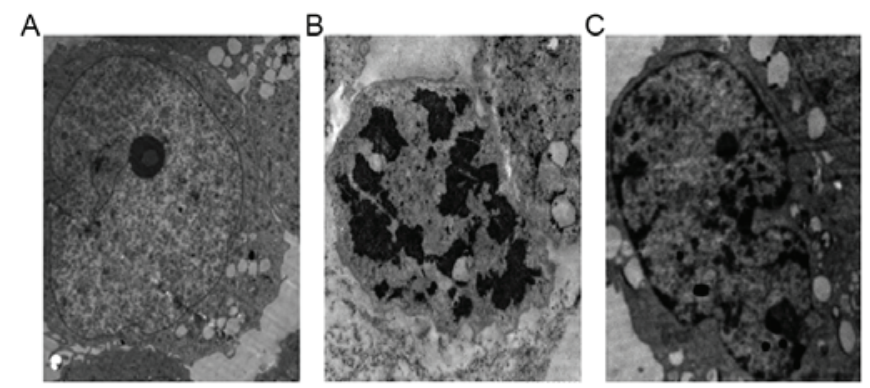

Figure 2. Atorvastatin alleviates iohexol-induced HK-2 cell morphological changes. Transmission electron microscopy images of (A) group A, (B) group B and (C) group C3. Magnification, x3.0 k.

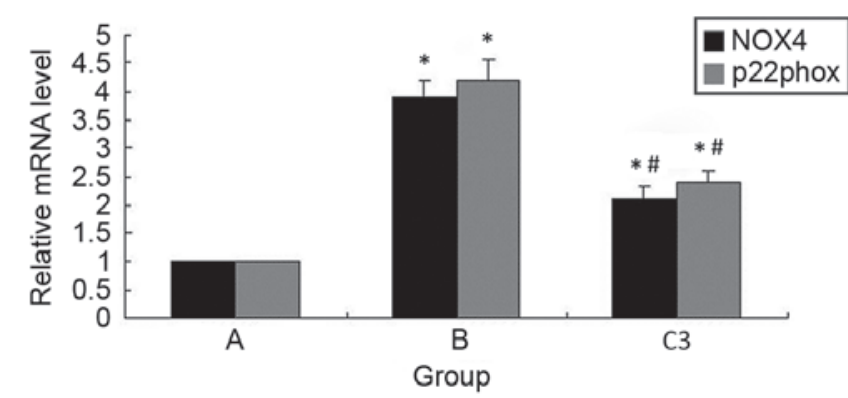

Figure 3. Atorvastatin inhibits iohexol-induced NOX subunit upregulation in HK-2 cells. " $\mathrm{P}<0.05$ vs. group $\mathrm{A} ;{ }^{*} \mathrm{P}<0.05$ vs. group B. p22phox, p22 phagocyte B-cytochrome; NOX, nicotinamide adenine dinucleotide phosphate oxidase.

manner. However, no effective drug has been approved for the prevention of CIN, with hydration therapy remaining the primary preventative treatment.

Statins, traditional drugs for regulating cholesterol levels, have been demonstrated to possess pleiotropic effects that include the enhancement of endothelial nitric oxide production (18), anti-inflammatory and antioxidative actions $(19,20)$. Accumulating clinical evidence suggests that atorvastatin has a preventive effect against CIN, and can reduce the incidence of dialysis and long-term mortality in patients with $\operatorname{CIN}(7,8,11,21)$. However, another previous study has revealed that statin use prior to percutaneous coronary intervention is not associated with a reduction in CIN (22). Therefore, the present study aimed to investigate the effects and potential underlying molecular mechanisms of atorvastatin on iodinated contrast agent-induced cytotoxicity. This revealed that pretreatment with atorvastatin $(40 \mu \mathrm{M})$ for $2 \mathrm{~h}$ significantly attenuated the suppression of cell viability by iohexol in a dose-dependent manner. In addition, atorvastatin significantly decreased iohexol-induced HK-2 cell apoptosis. Furthermore, the morphological changes characteristic of apoptosis in the cell injury group were alleviated by atorvastatin pretreatment.

Previous studies have demonstrated that the pathogenesis of CIN is associated with ischemia, hypoxia, oxidative stress and direct toxicity (23). A previous study by our group indicated that NOX and oxidative stress serve an important role in CIN (24). Inhibition of NOX with apocynin has been identified to ameliorate renal blood flow, renal vascular resistance and reduce the number of infiltrating immune cells (25). Recently, components of the NOX system, including NOX4 and p22phox, 
were identified to be present in the kidney and associated with the overproduction of ROS (26). These results suggest that iodinated CM may upregulate NOX4 expression, increase intracellular ROS production and lead to CIN. The present study identified that iohexol significantly increased the expression of NOX4 and p22phox, while atorvastatin alleviated this upregulation. These data indicate that atorvastatin alleviates iohexol-induced cytotoxicity through downregulating intracellular NOX4 and p22phox mRNA expression.

In conclusion, the results of the present study suggest that atorvastatin alleviates the cytotoxicity induced by iohexol in HK-2 cells. In addition, the underlying molecular mechanism for this effect is associated with the inhibition of the apoptosis, and the downregulation of NOX4 and p22phox. These results indicate that atorvastatin is a potential therapeutic agent for the prevention of CIN and required further study.

\section{Acknowledgements}

The present study was supported by the National Natural Science Foundation of China (grant no.81570618), the Scientific Foundation of Hunan Province (grant nos. 2010FJ6008 and 2008JT3005) and Guangzhou Nucien Pharmaceutical Co., Ltd. The authors would like to thank Dr Yi-Ya Zhang (Key Laboratory of Protein Chemistry and Developmental Biology of the Ministry of Education, College of Life Sciences, Hunan Normal University, Changsha, China) for reviewing the manuscript.

\section{References}

1. Aydoğdu S: Contrast-induced nephropathy. Turk Kardiyol Dern Ars 41: 28-30, 2013.

2. Jorgensen AL: Contrast-induced nephropathy: Pathophysiology and preventive strategies. Crit Care Nurse 33: 37-46, 2013.

3. Maliborski A, Zukowski P, Nowicki G and Bogusławska R: Contrast-induced nephropathy-a review of current literature and guidelines. Med Sci Monit 17: RA199-RA204, 2011.

4. Liang S, Kisseleva T and Brenner DA: The role of NADPH oxidases (NOXs) in liver fibrosis and the activation of Myofibroblasts. Front Physiol 7: 17, 2016.

5. Gorin Y and Wauquier F: Upstream regulators and downstream effectors of NADPH oxidases as novel therapeutic targets for diabetic kidney disease. Mol Cells 38: 285-296, 2015.

6. Qiu Y, Tao L, Lei C, Wang J, Yang P, Li Q and Lei B: Downregulating p22phox ameliorates inflammatory response in Angiotensin II-induced oxidative stress by regulating MAPK and NF-kB pathways in ARPE-19 cells. Sci Rep 5: 14362, 2015.

7. Li Y, Liu Y, Fu L, Mei C and Dai B: Efficacy of short-term high-dose statin in preventing contrast-induced nephropathy: A meta-analysis of seven randomized controlled trials. PLoS One 7: e34450, 2012.

8. Zhang T, Shen LH, Hu LH and He B: Statins for the prevention of contrast-induced nephropathy: A systematic review and meta-analysis. Am J Nephrol 33: 344-351, 2011.

9. Patti G, Nusca A, Chello M, Pasceri V, D Ambrosio A, Vetrovec GW and Di Sciascio G: Usefulness of statin pretreatment to prevent contrast-induced nephropathy and to improve long-term outcome in patients undergoing percutaneous coronary intervention. Am J Cardiol 101: 279-285, 2008.
10. Sadat U: Contrast-induced nephropathy: Do statins offer protection? Curr Opin Cardiol 26: 334-337, 2011.

11. Patti G, Ricottini E, Nusca A, Colonna G, Pasceri V, D Ambrosio A, Montinaro A and Di Sciascio G: Short-term, high-dose Atorvastatin pretreatment to prevent contrast-induced nephropathy in patients with acute coronary syndromes undergoing percutaneous coronary intervention from the ARMYDA-CIN (atorvastatin for reduction of myocardial damage during angioplasty-contrast-induced nephropathy) trial. Am J Cardiol 108: 1-7, 2011.

12. Alvarez E, Rodiño-Janeiro BK, Ucieda-Somoza R and González-Juanatey JR: Pravastatin counteracts angiotensin II-induced upregulation and activation of NADPH oxidase at plasma membrane of human endothelial cells. J Cardiovasc Pharmacol 55: 203-212, 2010.

13. Sarath TS, Waghe P, Gupta P, Choudhury S, Kannan K, Pillai AH, Harikumar SK, Mishra SK and Sarkar SN: Atorvastatin ameliorates arsenic-induced hypertension and enhancement of vascular redox signaling in rats. Toxicol Appl Pharmacol 280: 443-454, 2014.

14. Jo SH, Koo BK, Park JS, Kang HJ, Cho YS, Kim YJ, Youn TJ, Chung WY, Chae IH, Choi DJ, et al: Prevention of radiocontrast medium-induced nephropathy using short-term high-dose simvastatin in patients with renal insufficiency undergoing coronary angiography (PROMISS) trial-a randomized controlled study. Am Heart J 155: 499.e1-8, 2008.

15. Livak KJ and Schmittgen TD: Analysis of relative gene expression data using real-time quantitative PCR and the 2(-Delta Delta C(T)) Method. Methods 25: 402-408, 2001.

16. Stacul F, van der Molen AJ, Reimer P, Webb JA, Thomsen HS, Morcos SK, Almén T, Aspelin P, Bellin MF and Clement O: Contrast induced nephropathy: Updated ESUR contrast media safety committee guidelines. Eur Radiol 21: 2527-2541, 2011

17. Duan S, Zhou X, Liu F, Peng Y, Chen Y, Pei Y, Ling G, Zhou L, Li Y, Pi Y, et al: Comparative cytotoxicity of high-osmolar and low-osmolar contrast media on HKCs in vitro. J Nephrol 19: 717-724, 2006.

18. Gattone M: Measurement of C-reactive protein for the targeting of statin therapy in the primary prevention of acute coronary events. Ital Heart J Suppl 2: 1133-1134, 2001 (In Italian).

19. Ma W, Shen D, Liu J, Pan J, Yu L, Shi W, Deng L, Zhu L, Yang F, Liu J, et al: Statin function as an anti-inflammation therapy for depression in patients with coronary artery disease by downregulating interleukin-1 $\beta$. J Cardiovasc Pharmacol 67: 129-135, 2016.

20. Jaikumkao K, Pongchaidecha A, Chattipakorn N, Chatsudthipong V, Promsan S, Arjinajarn P and Lungkaphin A: Atorvastatin improves renal organic anion transporter 3 and renal function in gentamicin-induced nephrotoxicity in rats. Exp Physiol 101: 743-753, 2016.

21. John S, Schneider MP, Delles C, Jacobi J and Schmieder RE: Lipid-independent effects of statins on endothelial function and bioavailability of nitric oxide in hypercholesterolemic patients. Am Heart J 149: 473, 2005.

22. Kandula P, Shah R, Singh N, Markwell SJ, Bhensdadia N and Navaneethan SD: Statins for prevention of contrast-induced nephropathy in patients undergoing non-emergent percutaneous coronary intervention. Nephrology (Carlton) 15: 165-170, 2010.

23. Wong PC, Li Z, Guo J and Zhang A: Pathophysiology of contrast-induced nephropathy. Int J Cardiol 158: 186-192, 2012.

24. Yang SK, Duan SB, Pan P, XU XQ, Liu N and Xu J: Preventive effect of pentoxifylline on contrast-induced acute kidney injury in hypercholesterolemic rats. Exp Ther Med 9: 384-388, 2015.

25. Basile DP, Leonard EC, Beal AG, Schleuter D and Friedrich J: Persistent oxidative stress following renal ischemia-reperfusion injury increases ANGII hemodynamic and fibrotic activity. Am J Physiol Renal Physiol 302: F1494-F1502, 2012.

26. Choi HI, Ma SK, Bae EH, Lee J and Kim SW: Peroxiredoxin 5 protects TGF- $\beta$ induced fibrosis by inhibiting Stat 3 activation in rat kidney interstitial fibroblast cells. PLoS One 11: e0149266, 2016. 